\title{
ORTHOGONAL AND PRINCIPAL COMPONENTS ANALYSIS OF GENOTYPE-ENVIRONMENTAL INTERACTIONS FOR MULTIPLE METRICAL TRAITS
}

\author{
JEAN M. PERKINS \\ Department of Genetics, The University of Birmingham, P.O. Box 363, Birmingham B15 2TT
}

Received 20.iii.73

\begin{abstract}
Summary
In a previous paper Perkins and Jinks (1973) found that most of the genotypeenvironmental interactions of a set of inbred lines (derived by nine successive generations of selfing from a random sample of $\mathrm{F}_{2}$ individuals of the cross between two inbred varieties, 1 and 5 , of Nicotiana rustica) were linearly related to the dependent environmental component, $\hat{\epsilon}_{j}$ (Freeman and Perkins, 1973), for the four traits flowering time, linear growth rate, leaf length and final plant height in each of two experiments. The first experiment consisted of the complete set of 82 inbred genotypes, grown in the eight environments produced by two planting densities in each of four sowing dates and the second experiment consisted of a subsample of 10 inbred genotypes, selected as a stratified sample for mean performance in final plant height, grown in the 16 environments produced by all combinations of the presence and absence of $\mathrm{N}, \mathrm{P}, \mathrm{K}$ and $\mathrm{Ca}$ fertilisers. Each genotype was represented in each environment by eight individually randomised plants. In the present paper the linear interactions observed for experiment 1 were found to be primarily due to the differential linear, quadratic or cubic responses of the genotypes to successive (equidistant) sowing dates. Those of experiment 2 could be mostly attributed to one of the three main fertiliser treatments $\mathrm{N}, \mathrm{P}$ or $\mathrm{K}$ although the particular treatment responsible varied from trait to trait and according to the absence or presence of Ca.

A principal components analysis was applied to the correlation matrix between the orthogonal genotypic and $g \times e$ interaction comparisons of all the traits over genotypes in the two experiments. For experiment $l$ this showed that the $g \times e$ interaction comparison of each trait responsible for the linear relationship with $\hat{\epsilon}_{j}$ was sometimes associated in a complex manner with the genotypic component of a different trait but not significantly with that of the same trait. For experiment 2 the expected convergence of the quantitative traits in a poorer range of environments (this experiment was also grown at high density) was confirmed since most of the genotypic and important $g \times e$ interaction comparisons were found to be positively associated with the first principal component in both the absence and presence of calcium. A principal components analysis was similarly applied to the genotypic and density interaction comparisons of a subset of data including four further characters, capsule length, capsule width, capsule number and total seed yield collected from sowing 1, experiment 1 . This showed a positive association between total seed yield (a measure of fitness) and a linear growth rate, leaf length, final plant height, capsule length and capsule number. The interaction of seed yield with densities together with that of several other traits was found to be negatively associated with the genotypic component of flowering time due to the retardation in certain developmental processes of the earlier flowering genotypes at the higher planting density.
\end{abstract}

\section{INTRODUGTION}

IN a previous paper (Perkins and Jinks, 1973) the interactions of inbred lines of Nicotiana rustica, derived from crossing the two true breeding varieties 1 
and 5, with a number of different environmental variables was investigated. A significant proportion of the variation due to the interaction of genotypes and environments was observed to be linearly related to the additive environmental values for four metrical traits in most, if not all, the kinds of environments and in one experiment it was shown that the extent of this linearity was the same irrespective of whether the dependent or any one of several independent assessments of the environmental values was used (Freeman and Perkins, 1971).

The experimental designs used to generate the environments in each experiment permit an orthogonal breakdown of the genotype-environmental interactions into effects due to sowing dates, densities and their interaction in the larger of the two experiments and effects due to different combinations of the presence or absence of four fertiliser treatments (nitrogen, phosphorus, potassium and calcium) in the smaller, subsidiary experiment. One of the purposes of the present paper is to investigate which if any of these deliberately varied components of the environment are primarily responsible for the linear interactions already reported.

The interrelationships, as revealed by principal components analysis (see, for instance, Seal, 1964, and Perkins, 1972) of the four metrical traits, flowering time, growth rate, leaf length and final height, and of the interaction of these traits with environments will be discussed. The interrelationships of the same four metrical traits and four further characters, capsule length, capsule width, capsule number and seed yield which are components of "fitness", together with the interactions of all eight characters in two of the environments will also be examined. The value of relating genotypic performance for primary metrical traits to that for fitness characters is clearly demonstrated by the recent work of Linney, Barnes and Kearsey (1971) on the genetic architecture of sternopleural chaetae number in Drosophila melanogaster and of Westerman and Lawrence (1970) on linear genotype-environmental interactions which occur among inbred lines of Arabidopsis thaliana.

\section{EXPERIMENTAL DESIGNS AND METRICAL TRAITS}

Details of the experimental designs and of the characters scored for the two basic experiments are given by Perkins and Jinks (1973).

The genotypes of experiment 1 consisted of the 82 inbred lines each derived by nine successive generations of selfing from a single, randomly chosen, $\mathrm{F}_{2}$ plant of the cross between two true breeding varieties of $\mathcal{N}$. rustica, 1 and 5. These were grown in each of eight environments consisting of the eight combinations of two plant densities (normal, ND, and high, HD) on each of the four sowing dates (seed being sown into peat pots in the glasshouse and the plants, after germination, being hardened off in the cold frames before transplantation to the field, in the customary way, at two weekly invervals from April to early June).

The genotypes of experiment 2 consisted of a subset of 10 inbred lines (from among the 82) chosen as a stratified sample of mean performance for the character final plant height. These were grown in each of 16 experimental plots to which one of the 16 possible combinations of the presence or absence of the four fertiliser treatments nitrogen $(\mathrm{N})$, phosphorus $(\mathrm{P})$, potassium $(\mathrm{K})$, and calcium $(\mathrm{Ca})$, had been applied. 
Each inbred genotype in each environment of each experiment was represented by eight, individually randomised, replicate plants. All the plants in both experiments were scored for the four following metrical traits:

(i) Flowering time, FT, as days after sowing.

(ii) Linear growth rate, GR, in centimetres per day.

(iii) Leaf length, LL, of a fully expanded leaf (centimetres).

(iv) Final plant height, $\mathrm{FH}$ (centimetres).

For those plants grown in the normal and high densities of sowing 1, experiment 1 , the four additonal characters were also measured:

(v) Capsule length, GL, of a normal, randomly chosen capsule.

(vi) Capsule width, CW, of the same capsule as measured for CL.

(vii) Capsule number, CN.

(viii) Total seed yield, SY.

In order to estimate total seed yield (character viii) the seeds contained in ten ripe capsules of each plant were first counted on a "Decca Mastercount"

TABLE 1

The partitioning of the total degrees of freedom into items of a joint regression (a) and complete orthogonal $(b)$ analysis for experiment 1. See Section 3 for a detailed explanation

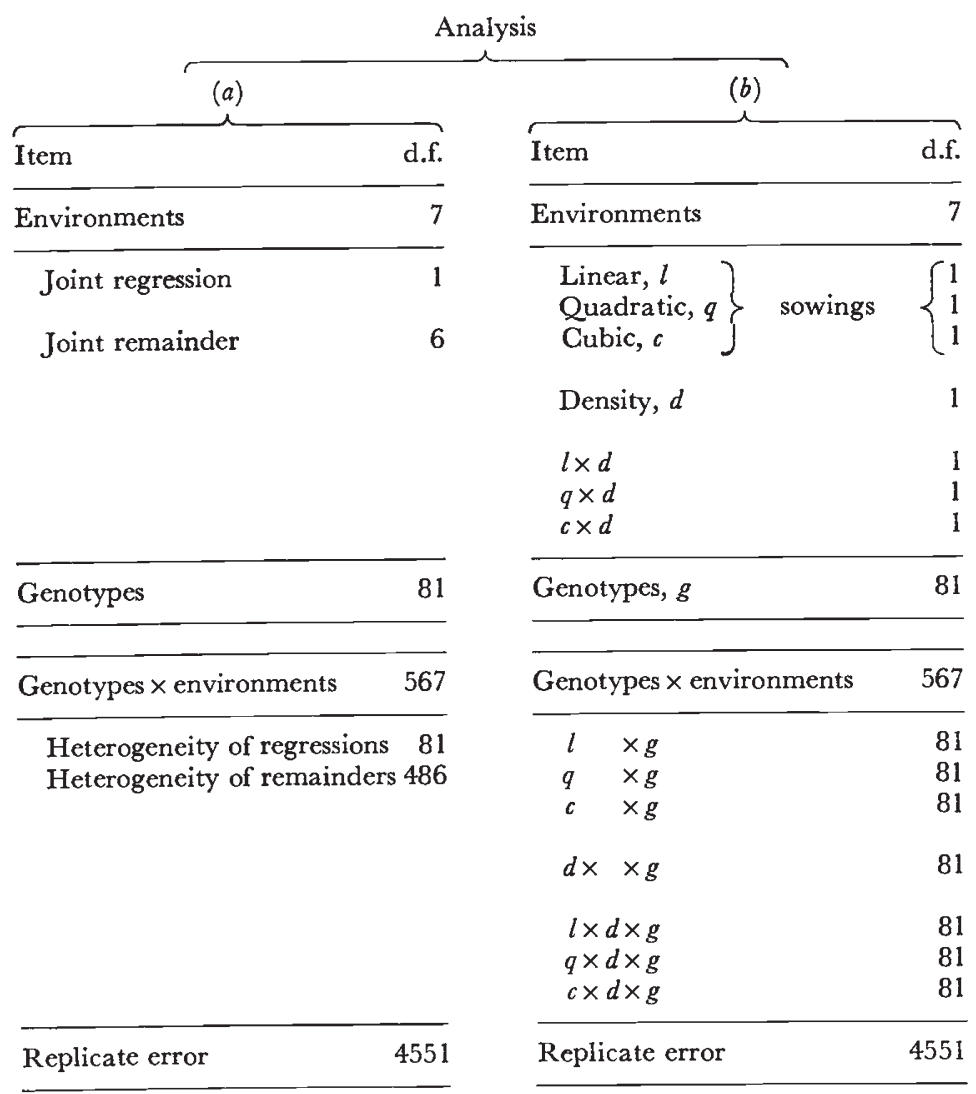


with an "Advance" batch counter. The average number of seeds per capsule could then be computed and when multiplied by the capsule number, CN, gave for each plant an estimate of total seed yield, SY.

TABLE 2

The partitioning of the total degrees of freedom into items of a joint regression $(a)$ and complete orthogonal $(b)$ analysis for either data subset (according to the absence or presence of calcium) of experiment 2. See Section 3 for a detailed explanation

Analysis

(a)

\begin{tabular}{lr}
$\overbrace{\text { Item }}$ & d.f. \\
\hline Environments & 7 \\
\hline Joint regression & 1 \\
Joint remainder & 6
\end{tabular}

\begin{tabular}{ll} 
Genotypes & 9 \\
\hline
\end{tabular}

Genotypes $\times$ environments $\quad 63$

Heterogeneity of regressions 9

Heterogeneity of remainders 54

$\begin{array}{ll}\overline{\text { Replicate error }}-\mathrm{Ca} & 560 \\ +\mathrm{Ca} & 559\end{array}$

(b)

\begin{tabular}{|c|c|}
\hline Item & d.f. \\
\hline Environments & 7 \\
\hline \multirow{3}{*}{$\begin{array}{l}\text { Nitrogen, } n \\
\text { Phosphorus, } p \\
\text { Potassium, } k\end{array}$} & 1 \\
\hline & 1 \\
\hline & 1 \\
\hline$n \times p$ & 1 \\
\hline \multirow{2}{*}{$\begin{array}{r}\quad \times k \\
p \times k\end{array}$} & 1 \\
\hline & 1 \\
\hline$n \times p \times k$ & 1 \\
\hline Genotypes, $g$ & 9 \\
\hline Genotypes $\times$ environments & 63 \\
\hline \multirow{3}{*}{$\begin{aligned} n & \times g \\
& \times g \\
& k \times g\end{aligned}$} & 9 \\
\hline & 9 \\
\hline & 9 \\
\hline$n \times p$ & 9 \\
\hline \multirow{2}{*}{$\begin{array}{r}\times k \times g \\
p \times k \times g\end{array}$} & 9 \\
\hline & 9 \\
\hline$n \times p \times k \times g$ & 9 \\
\hline \multirow{2}{*}{$\begin{aligned} \text { Replicate error } & -\mathrm{Ca} \\
& +\mathrm{Ca}\end{aligned}$} & 560 \\
\hline & 559 \\
\hline
\end{tabular}

\section{Analytical procedures}

The degrees of freedom, d.f., available for genotypes, $g$, environments, $e$, and genotype-environmental interactions, $g \times e$, were partitioned by Perkins and Jinks (1973) for both experiments into items appropriate for a joint regression analysis against the dependent environmental component, $\hat{\epsilon}_{j}$ (Perkins and Jinks, 1968; Freeman and Perkins, 1971). This method merely relies upon the empirical observation that interactions are often linearly related to the biological measure of the environment, $\hat{\epsilon}_{j}$, estimated from the data iself and in no way depends upon a knowledge of the physical factors varying from one environment to another. Due to the orthogonal 
(cross-classified) design of the environments in the two experiments, however, it is possible to repartition the degrees of freedom for environments and $g \times e$ interactions into further items due to specific, deliberately varied, environmental factors (the sowing date and density of experiment 1 and fertiliser treatments in experiment 2) and their interactions. The two methods of partition are compared for experiment 1 in table 1 and for experiment 2 in table 2.

The seven degrees of freedom available for differences between environments in experiment $l$ have been partitioned into seven items each with one degree of freedom. These items are the linear, $l$, quadratic, $q$, and cubic, $c$, orthogonal polynomial comparisons (Fisher and Yates, 1963) which can be derived for differences between sowing dates (three degrees of freedom), the density, $d$, comparison and the three interactions between the linear, quadratic and cubic sowing date effects, respectively, and density $(l \times d$, $q \times d$ and $c \times d)$. The orthogonal coefficients ( $k$ values) required in each environment (where ND = normal density and $\mathrm{HD}=$ high density) for the correction term and each environmental comparison are as follows:

\begin{tabular}{|c|c|c|c|c|c|c|c|c|}
\hline \multirow[b]{3}{*}{ Comparison } & \multicolumn{8}{|c|}{ Sowing } \\
\hline & \multicolumn{2}{|c|}{1} & \multicolumn{2}{|c|}{2} & \multicolumn{2}{|c|}{3} & \multicolumn{2}{|c|}{4} \\
\hline & ND & HD & ND & $\mathrm{HD}$ & ND & $\mathrm{HD}$ & ND & $\mathrm{HD}$ \\
\hline Correction term & 1 & 1 & 1 & 1 & 1 & 1 & 1 & \\
\hline $\int l$ & -3 & -3 & -1 & -1 & 1 & 1 & 3 & 3 \\
\hline Sowings $\{q$ & 1 & 1 & -1 & -1 & -1 & -1 & 1 & 1 \\
\hline$c$ & -1 & -1 & 3 & 3 & -3 & -3 & 1 & 1 \\
\hline Densities, $d$ & 1 & -1 & 1 & -1 & 1 & -1 & 1 & -1 \\
\hline$l \times d$ & -3 & 3 & -1 & 1 & 1 & -1 & 3 & -3 \\
\hline$q \times d$ & 1 & -1 & -1 & 1 & -1 & 1 & 1 & -1 \\
\hline$c \times d$ & -1 & 1 & 3 & -3 & -3 & 3 & 1 & -1 \\
\hline
\end{tabular}

The 567 degrees of freedom available for genotype-environmental interactions have been partitioned into seven items, each with 81 degrees of freedom, corresponding to the seven interactions between the seven environmental components $(l, q, c, d, l \times d, q \times d$ and $c \times d)$ and the 82 genotypes $(g)$.

In experiment 2 , data collected from the eight environmental plots each treated with one of the eight combinations of the presence or absence of nitrogen, phosphorus and potassium have, as before (Perkins and Jinks, 1973), been analysed separately according to whether or not they had also been treated with calcium. The partitioning of the total degrees of freedom into those for the various component items is therefore identical in the two subsets of data, with the exception of the replicate error item (differences between individuals within genotypes and environments) which has 560 degrees of freedom in the absence of calcium and 559 in its presence. The seven degrees of freedom available for differences between environments have been partitioned into seven items each with one degree of freedom. These items are the seven orthogonal comparisons of nitrogen, $n$, phosphorus, $p$, and potassium, $k$, and their second order $(n \times p, n \times k$ and $p \times k)$ and third order $(n \times p \times k)$ interactions. The orthogonal coefficients required in each environment for the correction term and each environmental comparison are as follows: 


\begin{tabular}{|c|c|c|c|c|c|c|c|c|}
\hline \multirow[b]{2}{*}{ Comparison } & \multicolumn{8}{|c|}{ Fertiliser treatment $(+$ or $-\mathrm{Ca})$} \\
\hline & $\mathbf{N}$ & $\mathbf{P}$ & $\mathbf{K}$ & NP & NK & PK & NPK & $(\mathrm{Nil})$ \\
\hline Correction term & 1 & 1 & 1 & 1 & 1 & 1 & 1 & 1 \\
\hline & 1 & -1 & -1 & 1 & 1 & -1 & 1 & -1 \\
\hline$p$ & -1 & 1 & -1 & 1 & -1 & 1 & 1 & -1 \\
\hline$k$ & -1 & -1 & 1 & -1 & 1 & 1 & 1 & -1 \\
\hline$n \times p$ & -1 & -1 & 1 & 1 & -1 & -1 & 1 & 1 \\
\hline$n \times k$ & -1 & 1 & -1 & -1 & 1 & -1 & 1 & 1 \\
\hline$p \times k$ & 1 & -1 & -1 & -1 & -1 & 1 & 1 & 1 \\
\hline$n \times p \times k$ & 1 & 1 & 1 & -1 & -1 & -1 & 1 & -1 \\
\hline
\end{tabular}

The 63 degrees of freedom available for genotype-environmental interactions have been partitioned into seven items, each with nine degrees of freedom, corresponding to the seven interactions between the seven orthogonal combinations of $\mathrm{N}, \mathrm{P}$ and $\mathrm{K}(n, p, k, n \times p, n \times k, p \times k$ and $n \times p \times k)$ and ten genotypes $(g)$.

The results of the analyses of variance for the two experiments, after a complete orthogonal breakdown of the environmental and $g \times e$ interaction items (column $(b)$ of tables 1 and 2, respectively), are summarised in Section 4. Subsequent investigations to identify, first, those environmental factors mainly responsible for the linear interactions after regression against $\hat{\boldsymbol{\epsilon}}_{j}$ and, second, the principal components of genotypic performance and $g \times e$ interactions for the four primary and four fitness traits are reported in Sections 5 and 6 , respectively.

In order to carry out these subsequent analyses it is first necessary to compute a linear score for the mean performance over environments and for the interaction with each environmental component of each genotype in each of the two experiments for each metrical trait. For the four primary traits, FT, GR, LL and FH, the orthogonal coefficients or $k$ values of the correction term and each environmental comparison in the eight environments, as already given for each experiment, are required. For the data subset of the same four primary traits and the four fitness characters, GL, GW, GN and SY, scored on the replicate individuals of the 82 inbred lines in the normal (ND) and high (HD) densities of sowing 1 (experiment 1) the following coefficients are appropriate:

$\begin{array}{lcr}\text { Comparison } & \overbrace{\text { ND }}^{\text {Density }} & \text { HD } \\ \text { Correction term } & 1 & 1 \\ \text { Densities } & 1 & -1\end{array}$

In each case, the linear score of each comparison for each genotype was derived from the following general formula:

$$
\sum k_{j} y_{i j}^{\prime}
$$

where, for a particular comparison, $k_{j}$ is the orthogonal coefficient in the $j$ th environment and $y_{i j}^{\prime}$ is the deviation, in the $j$ th environment, of the mean of the $i$ th genotype (over the eight replicate individuals) from the mean of all genotypes. By substituting into this formula the coefficient values of the correction term and each environmental comparison, a linear score was obtained for the mean performance over environments and for the interaction with each environmental component, respectively, of each genotype for each metrical trait. 


\section{ORthogonal BREAKDOWN OF ENVIRONMENTAL AND $g \times e$ INTERACTION COMPONENTS OF VARIATION}

Perkins and Jinks (1973) showed that linear regression against the dependent environmental component, $\hat{\epsilon}_{j}$, accounted for a significantly greater proportion of the variation due to genotype-environmental interactions than did the deviations from regression for the four characters, FT, GR, LL and FH, in both experiments 1 and 2 with the exception of flowering

TABLE 3

The components of variance, $\hat{\sigma}$ 's, and their significance, $P$, for the four metrical traits $F T, G R, L L$ and $F H$ in experiment 1 . The abbreviations used to denote the characters and the items of the analysis are explained in Sections 2 and 3 , respectively

\begin{tabular}{|c|c|c|c|c|c|c|c|c|c|c|}
\hline \multirow{2}{*}{\multicolumn{3}{|c|}{$\overbrace{}^{\text {Item }}$}} & \\
\hline & & & \multicolumn{2}{|c|}{$\mathrm{FT}$} & \multicolumn{2}{|c|}{ GR } & \multicolumn{2}{|c|}{ LL } & \multicolumn{2}{|c|}{ FH } \\
\hline $\begin{array}{l}\text { Sowing } \\
\text { dates }\end{array}$ & Densities & Genotypes & $\hat{\sigma}^{\mathbf{2}}$ & $\vec{P}$ & $\hat{\sigma}^{2}$ & $P$ & $\hat{\sigma}^{2}$ & $\mathbf{P}$ & $\hat{\sigma}^{2}$ & $\vec{P}$ \\
\hline$l$ & & & $13 \cdot 19$ & $* * *$ & - & n.s. & $0 \cdot 16$ & $* * *$ & $209 \cdot 84$ & $* * *$ \\
\hline$q$ & & & 8.94 & $* * *$ & 0.278 & $* * *$ & $0 \cdot 14$ & $* * *$ & 93.79 & *** \\
\hline \multirow[t]{2}{*}{ c } & & & - & n.s. & 0.201 & $* * *$ & - & n.s. & $45 \cdot 42$ & $* * *$ \\
\hline & $d$ & & - & n.s. & 0.006 & $* * *$ & 0.03 & $* * *$ & $45 \cdot 86$ & $* * *$ \\
\hline$l$ & $\times d$ & & - & n.s. & $0 \cdot 036$ & $* * *$ & 0.08 & $* * *$ & $24 \cdot 89$ & $* * *$ \\
\hline$q$ & $\times d$ & & 0.46 & $* * *$ & 0.037 & $* * *$ & $0 \cdot 11$ & $* * *$ & 28.39 & $* * *$ \\
\hline \multirow[t]{2}{*}{ c } & $\times d$ & & 0.42 & $* *$ & $0 \cdot 016$ & $* * *$ & 0.42 & $* * *$ & - & n.s. \\
\hline & & $g$ & $34 \cdot 87$ & $* * *$ & 0.188 & $* * *$ & $2 \cdot 10$ & $* * *$ & $173 \cdot 72$ & $* * *$ \\
\hline$l$ & & $\times g$ & $4 \cdot 13$ & $* * *$ & 0.034 & $* * *$ & 0.29 & $* * *$ & 22.95 & $* * *$ \\
\hline$q$ & & $\times g$ & $2 \cdot 35$ & $* * *$ & 0.033 & $* * *$ & 0.20 & $* * *$ & $19 \cdot 36$ & *** \\
\hline \multirow[t]{2}{*}{$c$} & & $\times g$ & $2 \cdot 52$ & $* * *$ & 0.088 & $* * *$ & - & n.s. & $9 \cdot 95$ & $* * *$ \\
\hline & $d$ & $\times g$ & - & n.s. & 0.007 & $* * *$ & 一 & n.s. & $2 \cdot 49$ & $* * *$ \\
\hline$l$ & $\times d$ & $\times g$ & - & n.s. & 0.014 & $* *$ & - & n.s. & - & n.s. \\
\hline$q$ & $\times d$ & $\times g$ & - & n.s. & - & n.s. & - & n.s. & - & n.s. \\
\hline$c$ & $\times d$ & $\times g$ & $2 \cdot 09$ & *** & $0 \cdot 022$ & *** & - & n.s. & - & n.s. \\
\hline Repli & & & $21 \cdot 22$ & & $0 \cdot 232$ & & $4 \cdot 11$ & & $115 \cdot 49$ & \\
\hline
\end{tabular}

n.s. = Probability non-significant.

$*$ = Probability 0.01-0.05.** = Probability 0.001-0.01.***= Probability $<0.001$.

time in experiment 1 and leaf length in the presence of calcium in experiment 2. The linear regression coefficients, $\hat{\beta}_{d}$ 's, and genetical components, $[\hat{d}]$ 's, were in general uncorrelated but those of the 10 inbred genotypes of experiment 2 were more positively correlated in the relatively impoverished set of fertiliser treatments without calcium.

In the analysis of variance for experiment 1 , with a complete orthogonal breakdown (see column (b) of table 1), the environmental items for sowing dates and densities were treated as fixed effects whilst the item for genotypes was treated as a random effect, leading to a mixed model situation. The components of variance, $\hat{\sigma}^{2}$ 's, derived from the expectation of mean squares and their significance, $\mathrm{P}$, for the genotypic, environmental and $g \times e$ interaction items are given in table 3 for the four metrical traits, FT, GR, LL 
and FH. The values of the non-significant $\hat{\sigma}^{2}$ 's have been omitted from the table for clarity. It is obvious that, for all the characters, most of the variation due to environmental causes and their interactions with genotypes is accounted for by one or more of the sowing date effects (linear, quadratic or cubic).

In the analyses of variance for experiment 2 (in the absence or presence of calcium), with a complete orthogonal breakdown (see column (b) of table 2), the environmental items for the three fertiliser treatments, $N, P$

TABLE 4

The components of variance, $\hat{\sigma}^{2}$ 's, and their significance, $P$, for the four metrical traits $F T, G R, L L$ and $F H$ in the absence of calcium in experiment 2. The abbreviations used to denote the characters and the items of the analysis are explained in Sections 2 and 3, respectively

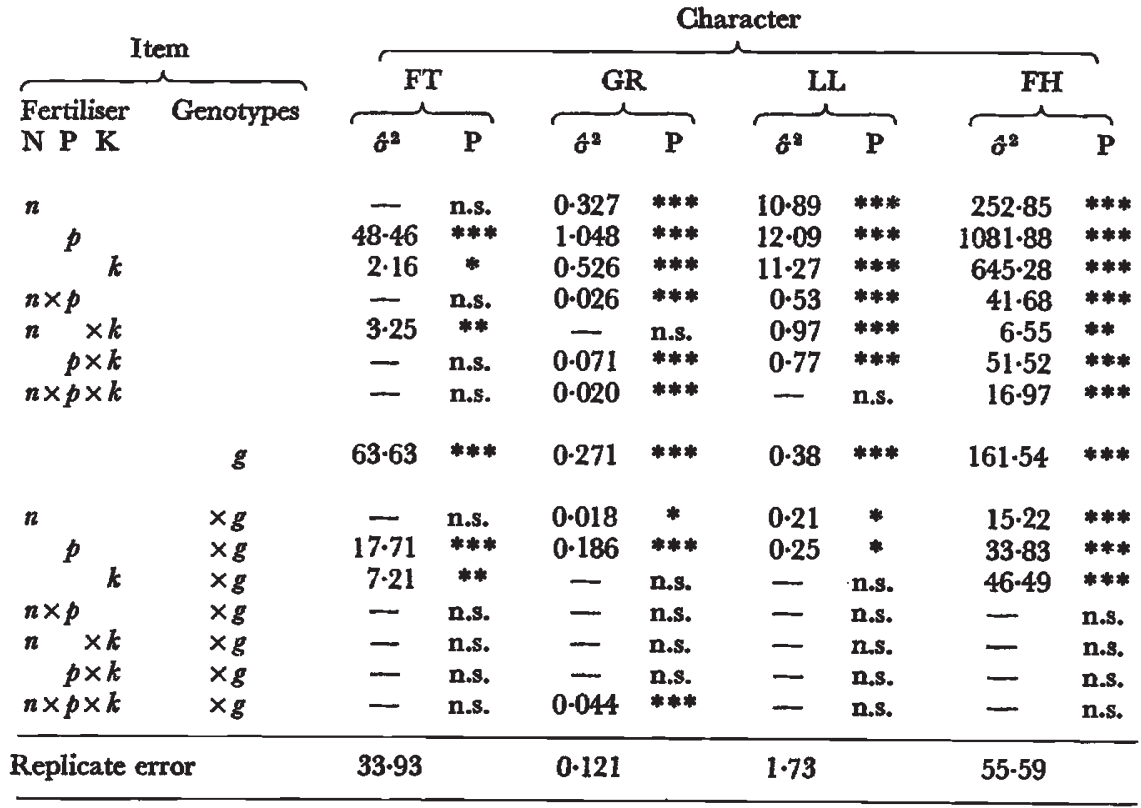

n.s., *,**,*** = Probability as in table 3.

and $\mathrm{K}$, and the item for genotypes have all been treated as fixed effects. The components of variance, $\hat{\sigma}^{2}$ 's and their significance, $P$, for the genotypic, environmental and $g \times e$ interaction items are given with calcium absent in table 4 and with calcium present in table 5 for the four metrical traits, FT, GR, LL and FH. The values of the non-significant $\hat{\sigma}^{2}$ 's have again been omitted from both tables. It is clear that, for all the characters, most of the variation due to environmental causes and their interactions with genotypes is accounted for by one or more of the three main fertiliser treatments, $\mathrm{N}, \mathrm{P}$ or $\mathrm{K}$, whether in the absence (table 4) or presence (table 5) of calcium. The interactions of leaf length in the presence of calcium are an exception since all are non-significant.

The four fitness characters CL, CW, GN and SY (see Section 2) scored on the eight replicate plants for each of the 82 inbred genotypes in the two densities (normal and high) of sowing 1, experiment 1, were each subjected to a simple two-way analysis of variance (treating genotypes as a random 
and densities as a fixed effect) with a replicate error item (from the average variance of individuals within genotypes and densities). All the items (genotypes, environments and the interaction of genotypes with environments) were highly significant for each of the characters except for the interaction

TABLE 5

The components of variance, $\hat{\sigma}^{2}$ 's, and their significance, $P$, for the four metrical traits $F T, G R, L L$ and $F H$ in the presence of calcium in experiment 2. The abbreviations used to denote the characters and the items of the analysis are explained in Sections 2 and 3, respectively

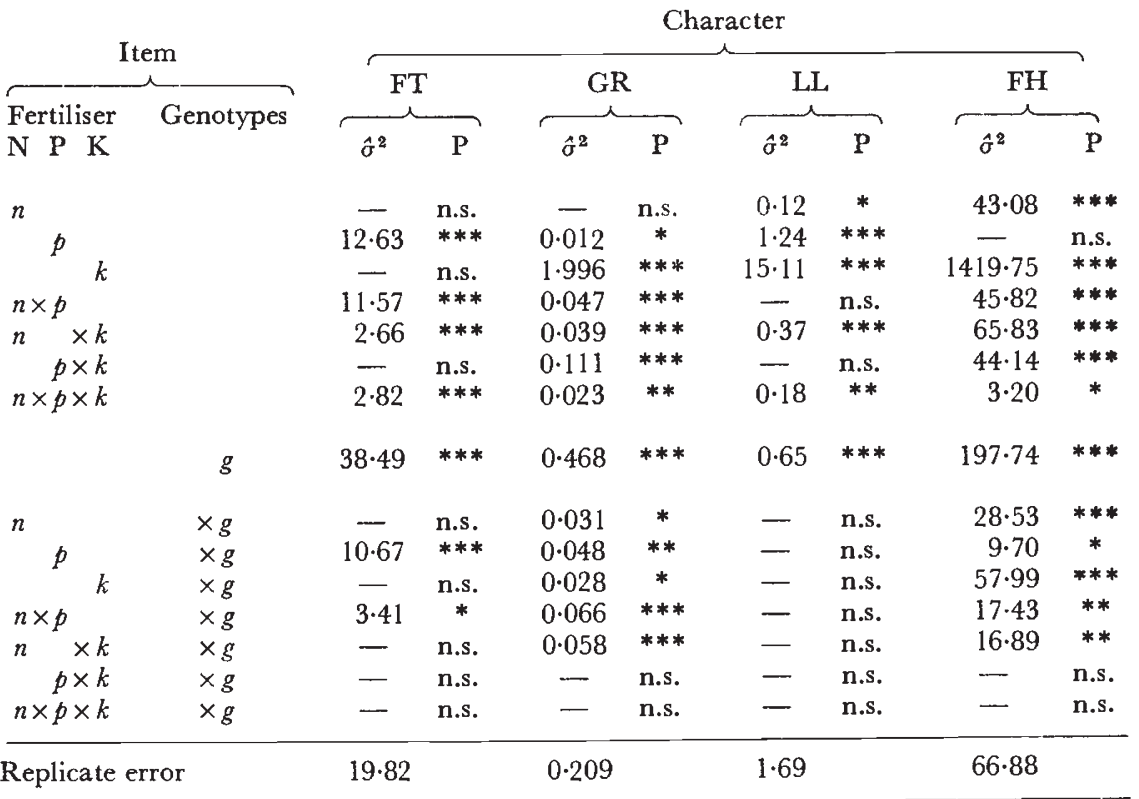

n.s., ${ }^{*}, * *, * * *=$ Probability as in table 3 .

items of capsule length and capsule width both of which just failed to reach significance at the 5 per cent level of probability.

\section{ENVIRONMENTAL FAGTORS RESPONSIBLE FOR THE LINEAR INTERAGTIONS OBTAINED AFTER REGRESSION AGAINST $\varepsilon_{j}$.}

For the four primary traits FT, GR, LL and FH in the two experiments 1 and 2 (the latter in both the absence and presence of calcium) the linear score for the interaction with each environmental component was derived in the manner described at the end of Section 3. Each of these interaction scores for each genotype was then correlated (over genotypes) with the corresponding linear regression coefficient, $\hat{\beta}_{d}$, of the genotype obtained from the regression of its interaction with each environment (without an orthogonal breakdown) against the dependent environmental component, $\varepsilon_{j}$. The correlation values (80 degrees of freedom) are given in table 6 for those genotype-environmental interaction comparisons of experiment 1 which have significant probabilities in table 3 . The correlation values (8 degrees of freedom) for the significant $g \times e$ interaction comparisons of 
experiment 2 (see tables 4 and 5) are given in table 7 (in both the absence and presence of calcium).

For each character in both experiments the interaction comparison which shows the highest correlation (irrespective of sign) with $\hat{\beta}_{d}$ (in table 6

TABLE 6

Correlation (over genotypes) between the linear score of each interaction comparison (see Section 3) and $\hat{\beta}_{d}$ for FT, GR, LL and FH (see Section 2) in experiment 1

\begin{tabular}{|c|c|c|c|c|c|}
\hline \multirow{2}{*}{\multicolumn{2}{|c|}{$\begin{array}{l}\text { Interaction } \\
\text { comparison }\end{array}$}} & \multicolumn{4}{|c|}{ Character } \\
\hline & & \multirow{2}{*}{$\begin{array}{c}\text { FT } \\
-0.71\end{array}$} & \multirow{2}{*}{$\begin{array}{c}\text { GR } \\
0.02\end{array}$} & \multirow{2}{*}{$\begin{array}{l}\text { LL } \\
0 \cdot 61\end{array}$} & \multirow{2}{*}{$\begin{array}{c}\text { FH } \\
0.89\end{array}$} \\
\hline$l$ & $\times g$ & & & & \\
\hline$q$ & $\times g$ & 0.22 & 0.79 & 0.62 & 0.50 \\
\hline$c$ & $\times g$ & 0.51 & -0.87 & - & -0.44 \\
\hline & $d \times g$ & - & 0.57 & - & 0.41 \\
\hline & $d \times g$ & - & -0.22 & - & - \\
\hline & $d \times g$ & - & - & - & - \\
\hline & $d \times g$ & $0 \cdot 19$ & -0.37 & - & - \\
\hline
\end{tabular}

\section{TABLE 7}

Correlation (over genotypes) between the linear score of each interaction comparison (see Section 3) and $\hat{\beta}_{d}$ for FT, GR, $L L$ and $F H$ (see Section 2) in experiment 2 (in both the absence and presence of calcium)

\begin{tabular}{|c|c|c|c|c|}
\hline \multirow{2}{*}{$\begin{array}{l}\text { Interaction } \\
\text { comparison }\end{array}$} & \multicolumn{4}{|c|}{ Character } \\
\hline & FT & GR & LL & $\mathrm{FH}$ \\
\hline \multicolumn{5}{|l|}{ Without $\mathrm{Ca}$} \\
\hline$n \quad \times g$ & - & 0.36 & -0.91 & 0.31 \\
\hline$\times \dot{g}$ & -0.98 & 0.97 & 0.78 & 0.94 \\
\hline$k \times g$ & -0.03 & - & - & 0.76 \\
\hline$n \times p \quad \times g$ & - & - & - & - \\
\hline$n \quad \times k \times g$ & - & - & - & - \\
\hline$p \times k \times g$ & - & - & - & - \\
\hline$n \times p \times k \times g$ & - & 0.65 & - & - \\
\hline \multicolumn{5}{|l|}{ With $\mathrm{Ca}$} \\
\hline$n \quad \times g$ & - & -0.23 & - & $0 \cdot 70$ \\
\hline$\times g$ & -0.96 & -0.14 & - & -0.69 \\
\hline$k \times g$ & - & 0.97 & - & 0.99 \\
\hline & -0.77 & -0.21 & - & -0.70 \\
\hline$n \times k \times g$ & - & 0.22 & 一 & 0.43 \\
\hline$p \times k \times g$ & - & - & - & - \\
\hline$n \times p \times k \times g$ & 一 & - & - & - \\
\hline
\end{tabular}

for experiment 1 and table 7 for experiment 2, calcium absent or present) usually has the largest or, where there is little difference, the next largest component of variance, $\hat{\sigma}^{2}$ (in table 3 for experiment 1 and tables 4 , calcium absent, and 5, calcium present, in experiment 2). The significant differences between genotypes for their $\beta_{d}$ values (based on the dependent environmental component, $\hat{\epsilon}_{j}$, Perkins and Jinks, 1973) for flowering time and final height in experiment 1 can be mainly attributed to the differences among the genotypes in their linear responses for these traits to successive (equidistant) 
sowing dates. The significant heterogeneity of regression item for leaf length is due equally to the differential linear and quadratic responses of the genotypes for this character to successive sowing dates while that for growth rate is primarily due to their differential quadratic and cubic responses. In experiment 2 one of the three main fertiliser treatments, nitrogen, phosphorus or potassium, is in general chiefly responsible for the differences observed among the genotypes for their $\beta_{d}$ values and the particular treatment responsible differs for some characters according to whether calcium is absent or present. In the absence of calcium it is the differential responses of the genotypes to the presence or absence of phosphorus which leads to the significant differences among the $\beta_{d}$ 's found for flowering time, growth rate and final height while for leaf length it is their differential responses to the presence or absence of nitrogen. In the presence of calcium the differences are due to the differential responses among the genotypes to the presence or absence of phosphorus for flowering time (as before) but the presence or absence of potassium for both growth rate and final height. No genotype $x$ environmental interactions were detected for leaf length in the presence of calcium in this experiment.

\section{Principal Gomponents analysis of the genotypic and $g \times e$ interaction COMPONENTS OF VARIATION AND GOVARIATION BETWEEN GENOTYPES FOR MULTIPLE METRICAL TRAITS}

The same basic analysis was applied to the four primary traits, FT, GR, $\mathrm{LL}$ and $\mathrm{FH}$, of the 82 genotypes in experiment 1 and the 10 genotypes in experiment 2 (the two data subsets, according to the absence or presence of calcium, have been analysed separately) and to the four primary and four fitness characters, CL, CW, CN and SY, of the 82 inbred lines in the normal and high densities of sowing 1 , experiment 1 . For each trait in each set of data the linear score was derived for the mean performance of each genotype over environments and for the interaction of each genotype with each orthogonal environmental component in the manner described at the end of Section 3. The linear scores of these variates were then correlated over genotypes in all possible pairwise combinations, both within and between traits, for each set of data. A principal components analysis was then applied to each correlation matrix. The correlation rather than sum of squares-sum of products or variance-covariance matrix was used since many of the characters were measured on different, unrelatable, scales. This has the disadvantage, however, that equal weighting is given to each genotypic component (mean performance over environments) and to each genotypeenvironmental interaction component of the total variance over genotypes even though they may differ considerably in their heritabilities (since the variance of each component is not corrected for its error) and in the magnitudes of their components of variance, $\hat{\sigma}^{2}$ 's (tables 3,4 and 5).

For the four primary traits, FT, GR, LL and FH, in experiment 1 the correlations over genotypes are given in table 8 between the score of each genotype on each of the first four principal components and their corresponding scores on each of the 32 original variates (the genotypic and seven $g \times e$ interaction components for each of the four traits). Only the first four components are easily interpretable and they jointly account for 45 per cent of the total variance of all the components (32 in all) over genotypes. In 
order to facilitate the interpretation of each component in table 8 their correlation with each genotype-environmental interaction comparison shown

TABLE 8

The correlations over genotypes for experiment 1 between the score of each genotype on each of the first four principal components and their corresponding scores on each of the 32 original variates, i.e. the genotypic and seven $g \times e$ interaction components of each of the four traits, FT, GR, LL and FH (see Sections 2, 3 and 6)

\begin{tabular}{|c|c|c|c|c|c|}
\hline \multirow{2}{*}{$\begin{array}{l}\text { Principal } \\
\text { component }\end{array}$} & \multirow{2}{*}{$\begin{array}{l}\text { Orthogonal } \\
\text { comparison }\end{array}$} & \multicolumn{4}{|c|}{ Character } \\
\hline & & FT & GR & LL & FH \\
\hline$\frac{1}{19 \% \dagger}$ & $\begin{array}{rr} & g \\
l & \times g \\
q & \times g \\
c & \times g \\
& d \times g \\
l \times d \times g \\
q \times d \times g \\
q \times d \times g \\
c \times d \times g\end{array}$ & $\begin{array}{c}0.87 \\
0.51 \\
-0.10_{+}^{+} \\
-0.54 \\
(0.20) \\
(0.02) \\
(-0.39) \\
-0.46\end{array}$ & $\begin{array}{r}0.05 \\
-0.04 \\
-0.66 \\
0.77 \\
-0.73 \\
0.14 \\
(0.50) \\
0.66\end{array}$ & $\begin{array}{r}0.22 \\
-0.20 \\
0.07 \\
(0.14) \\
(-0.20) \\
(0.21) \\
(0.03) \\
(0.30)\end{array}$ & $\begin{array}{r}0.68 \\
-0.03 \\
-0.68 \\
-0.43 \\
0.54 \\
(-0.21) \\
(0.27) \\
(0.53)\end{array}$ \\
\hline $\begin{array}{c}2 \\
11 \%\end{array}$ & $\begin{array}{rr} & g \\
l & \times g \\
q & \times g \\
c & \times g \\
& d \times g \\
l \times d \times g \\
q \times d \times g \\
q \times d \times g\end{array}$ & $\begin{array}{c}0.09 \\
0.27 \\
0.50 \\
0.30 \\
(-0.09) \\
(-0.01) \\
(-0.01) \\
0.13\end{array}$ & $\begin{array}{r}0.68 \\
0.62 \\
-0.09 \\
-0.31 \\
0.01 \\
-0.37 \\
(0.08) \\
0.05\end{array}$ & $\begin{array}{c}0.44 \\
0.42 \\
0.41 \\
(0.07) \\
(-0.02) \\
(-0.21) \\
(-0.02) \\
(-0.34)\end{array}$ & $\begin{array}{r}0.38 \\
0.76 \\
0.35 \\
-0.52 \\
0.28 \\
(-0.25) \\
(-0.21) \\
(-0.09)\end{array}$ \\
\hline $\begin{array}{l}3 \\
8 \%\end{array}$ & $\begin{array}{rr} & g \\
l & \times g \\
q & \times g \\
c & \times g \\
& d \times g \\
l \times d \times g \\
q \times d \times g \\
c \times d \times g\end{array}$ & $\begin{array}{c}0.14 \\
0.52 \\
0.66 \\
0.25 \\
(-0.54) \\
(-0.57) \\
(-0.23) \\
0.38\end{array}$ & $\begin{array}{r}-0.29 \\
0.18 \\
-0.25 \\
-0.04 \\
0.05 \\
0.36 \\
(0.10) \\
-0.05\end{array}$ & $\begin{array}{c}-0.37 \\
-0.04 \\
0.11 \\
(0.05) \\
(0.26) \\
(0.24) \\
(0.00) \\
(0.25)\end{array}$ & $\begin{array}{r}-0.29 \\
0.19 \\
0.01 \\
0.16 \\
-0.18 \\
(0.21) \\
(0.10) \\
(0.44)\end{array}$ \\
\hline $\begin{array}{l}4 \\
7 \%\end{array}$ & $\begin{array}{rr} & g \\
l & \times g \\
q & \times g \\
c & \times g \\
& d \times g \\
l \times d \times g \\
q \times d \times g \\
c \times d \times g\end{array}$ & $\begin{array}{r}-0.09 \\
-0.19 \\
0.04 \\
0.32 \\
(-0.24) \\
(-0.20) \\
(0.05) \\
0.07\end{array}$ & $\begin{array}{r}0.50 \\
-0.27 \\
-0.22 \\
-0.06 \\
0.26 \\
0.48 \\
(0.13) \\
-0.07\end{array}$ & $\begin{array}{r}0.62 \\
-0.39 \\
-0.03 \\
(0.04) \\
(-0.02) \\
(0.25) \\
(0.38) \\
(-0.30)\end{array}$ & $\begin{array}{r}0.37 \\
-0.26 \\
-0.11 \\
0.11 \\
0.09 \\
(0.47) \\
(0.23) \\
(-0.14)\end{array}$ \\
\hline
\end{tabular}

† The percentage value given represents the proportion of the total variance of all the principal components over genotypes which that particular component accounts for.

$\ddagger$ Correlation values less than $|0.5|$ are printed in italics.

( ) The correlation value of each component with each $g \times e$ interaction comparison shown to be non-significant in table 3 for each character has been placed in parentheses.

to be non-significant in table 3 for each character has been placed in parentheses and all the correlations below the arbitrary value of $|0.5|$ are printed in italics.

The first component shows a high positive correlation both with the genotypic component of flowering time and with the interaction between the cubic effect of sowing dates and genotypes for growth rate. Examination 
of the original mean performances in each environment for each trait of those genotypes with an extreme score on this component show that they either consistently flower late and grow faster in the second sowing relative to the third (see Section 3 for the derivation of the orthogonal cubic comparison for sowing dates) or they consistently flower early and grow slower in the second sowing relative to the third. It is of interest to note that for growth rate it was interaction between the cubic effect of sowing dates and genotypes which, as concluded in Section 5, accounted for most of the heterogeneity of regressions of this trait against the dependent environmental component, $\hat{\epsilon}_{j}$. The first principal component is also fairly highly correlated with several other comparisons including the genotypic component of final height.

The second principal component shows a high positive correlation both with the interaction between the linear effect of sowing dates and genotypes for final height and with the genotypic component of growth rate. Genotypes with an extreme score on this component either grow taller the later they are sown and have a high average growth rate or they show little response to successive sowing dates for final height and have a low average growth rate over environments. It was the differences among the genotypes in their linear responses to successive sowing dates for final height that accounted for most of the heterogeneity of regressions of this trait against the dependent environmental component, $\hat{\epsilon}_{j}$ (see Section 5). The third principal component is exclusively correlated with many of the interaction comparisons for flowering time even though some of these were found to be non-significant in table 3. Genotypes with an extreme score on this component either show little linear and a concave quadratic response to successive sowing dates for flowering time or they show a high linear (flowering earlier the later they are sown) and a convex quadratic response to successive sowing dates for this trait. The fourth component is only fairly highly correlated (in a positive direction) with the genotypic component of leaf length meaning that genotypes with an extreme score for this component either have a high or a low average leaf length over environments.

For the four primary traits, FT, GR, LL and FH, in experiment 2 the correlations over genotypes are given in table 9 between the score of each genotype on the first principal component and their corresponding scores on each of the 32 original variates (the genotypic and seven $g \times e$ interaction components for each of four traits) in both the absence and presence of calcium. As in table 8, the correlation of each genotype-environmental interaction comparison shown to be non-significant in table 4 or 5 for each character has been placed in parentheses and all the correlations below the arbitrary value of $|0.5|$ are printed in italics. Only nine principal components in total could be derived for each data subset (calcium absent or present) since the number of observations ( 10 genotypes) available for each variate is less than the total number of variates (32). Under these circumstances the number of components that can be extracted is equal to the number of observations minus one, i.e. nine. The first seven principal components jointly account for 96 per cent, in both data subsets, of the total variance of all the components over genotypes while the first component accounts for 33 per cent in the absence of calcium and 36 per cent in the presence of calcium of the total variance. Apart from the first principal component, however, the remaining six are usually only correlated with one 
or two of the genotypic or $g \times e$ interaction comparisons of the traits shown to be significant in tables 4 or 5 . The first principal component is highly correlated with the genotypic component of each of the three traits flowering time, growth rate and final height in both the absence and presence of calcium. It is also fairly highly correlated with the interaction component of each trait shown in Section 5 to be primarily responsible for its significant

TABLE 9

The correlations over genotypes for experiment 2, in both the absence and presence of calcium, between the score of each genotype on the first principal component and their corresponding scores on each of the 32 original variates, i.e. the genotypic and $g \times e$ interaction components of each of the four traits FT, GR, LL and FH (see Sections 2, 3 and 6 )

\begin{tabular}{|c|c|c|c|c|c|}
\hline \multirow[b]{2}{*}{ Calcium } & \multirow{2}{*}{$\begin{array}{l}\text { Orthogonal } \\
\text { comparison }\end{array}$} & \multicolumn{4}{|c|}{ Character } \\
\hline & & FT & GR & LL & FH \\
\hline \multirow{8}{*}{$\begin{array}{c}\text { Absent } \\
33 \% \dagger\end{array}$} & $g$ & $0 \cdot 85$ & $0 \cdot 85$ & 0.43 & $0 \cdot 91$ \\
\hline & $\times g$ & $(-0.03)$ & $0 \cdot 59$ & 0.64 & $0 \cdot 69$ \\
\hline & $\times g$ & $-0.84_{t}^{+}$ & $0 \cdot 86$ & $-0 \cdot 39$ & $0 \cdot 58$ \\
\hline & $k \times g$ & -0.23 & $(0.57)$ & $(0.04)$ & $0 \cdot 90$ \\
\hline & $n \times p \quad \times g$ & $(0 \cdot 28)$ & $(-0.42)$ & $(0.42)$ & $(0 \cdot 75)$ \\
\hline & $n \times k \times g$ & $(0 \cdot 88)$ & $(-0.21)$ & $(0.15)$ & $(0.35)$ \\
\hline & $p \times k \times g$ & $(0.23)$ & $(0.31)$ & $(0.42)$ & $(0.01)$ \\
\hline & $n \times p \times k \times g$ & $(-0.32)$ & 0.86 & $(0 \cdot 26)$ & $(0.72)$ \\
\hline \multirow{8}{*}{$\begin{array}{c}\text { Present } \\
36 \%\end{array}$} & $g$ & 0.97 & 0.93 & $(0.27)$ & 0.94 \\
\hline & $\times \dot{g}$ & $(0 \cdot 19)$ & $0 \cdot 18$ & $(0.40)$ & 0.85 \\
\hline & $\times g$ & -0.87 & $0 \cdot 63$ & $(-0.56)$ & -0.85 \\
\hline & $k \times g$ & $(0 \cdot 13)$ & $0 \cdot 13$ & $(-0 \cdot 34)$ & $0 \cdot 87$ \\
\hline & $n \times p \quad \times g$ & -0.81 & 0.59 & $(-0.12)$ & -0.58 \\
\hline & $n \times k \times g$ & $(-0.44)$ & $0 \cdot 69$ & $(-0.21)$ & 0.40 \\
\hline & $p \times k \times g$ & $(-0.15)$ & $(0 \cdot 72)$ & $(0.44)$ & $(0 \cdot 58)$ \\
\hline & $n \times p \times k \times g$ & $(0 \cdot 69)$ & $(0 \cdot 16)$ & $(-0 \cdot 76)$ & $(0.44)$ \\
\hline
\end{tabular}

$\dagger$ The percentage value given represents the proportion of the total variance of all the principal components over genotypes which that particular component accounts for.

$\$$ Correlation values less than $|0.5|$ are printed in italics.

( ) The correlation value of each $g \times e$ interaction comparison shown to be non-significant in table 3 for each character has been placed in parentheses.

heterogeneity of regressions against the dependent environmental component, $\hat{\epsilon}_{j}$, in either data subset with the exception of growth rate in the presence of calcium. The association of both the genotypic and the $g \times e$ interaction components of the traits with the first component is fully expected in both data subsets since the 10 inbred lines were chosen as a stratified sample of average final height over environments and the genotypic component, $[\hat{d}]$, and the corresponding linear regression coefficient, $\hat{\beta}_{d}$, of the interactions against $\hat{\epsilon}_{j}$ of the genotypes are expected to become more highly correlated in poorer sets of environments (this experiment was also sown at high density). It was only the seventh principal component in the absence of calcium and the fourth component in the presence of calcium which was highly correlated with the genotypic component of leaf length.

For the four primary (FT, GR, LL and FH) and four fitness (CL, CW, $\mathrm{CN}$ and $\mathrm{SY}$ ) traits from the two density levels of sowing l, experiment 1 , the correlations over genotypes are given in table 10 between the score of each genotype on each of the first five principal components and their correspond- 
ing scores on each of the 16 original variates (the genotypic and density $\times$ genotypes interaction component for each of the eight traits). All the correlations below the arbitrary value of $|0.5|$ in the table have been printed in italics. The first five principal components jointly account for 76 per cent of the total variance of all the components ( 16 in all) over genotypes. The

TABLE 10

The correlations over the 82 genotypes in the two density levels of sowing 1 , experiment 1 , between the score of each genotype on each of the first five principal components and their corresponding scores on each of the 16 original variates, i.e. the genotypic and density $\times$ genotype interaction components of each of the eight traits FT, GR, LL, FH, CL, CW, CN and SY (see Sections 2, 3 and 6)

\begin{tabular}{|c|c|c|c|c|c|c|}
\hline \multirow[b]{2}{*}{ Character } & \multirow{2}{*}{$\begin{array}{l}\text { Orthogonal } \\
\text { comparison }\end{array}$} & \multicolumn{5}{|c|}{ Principal component } \\
\hline & & 1 & 2 & 3 & 4 & 5 \\
\hline 1. FT & $\begin{array}{r}g \\
d \times g\end{array}$ & $\begin{array}{l}0.39 \dagger \\
0.07\end{array}$ & $\begin{array}{l}0.63 \\
0.09\end{array}$ & $\begin{array}{l}-0.18 \\
-0.37\end{array}$ & $\begin{array}{r}-0.15 \\
0.51\end{array}$ & $\begin{array}{r}-0.48 \\
0.63\end{array}$ \\
\hline 2. GR & $\begin{array}{r}g \\
d \times g\end{array}$ & $\begin{array}{r}0.58 \\
-0.08\end{array}$ & $\begin{array}{l}-0.46 \\
-0.61\end{array}$ & $\begin{array}{l}0.32 \\
0.12\end{array}$ & $\begin{array}{r}-0.03 \\
0.20\end{array}$ & $\begin{array}{r}0.22 \\
-0.50\end{array}$ \\
\hline 3. LL & $\begin{array}{r}g \\
d \times g\end{array}$ & $\begin{array}{l}0.78 \\
0.11\end{array}$ & $\begin{array}{r}0.12 \\
-0.54\end{array}$ & $\begin{array}{r}0.34 \\
-0.07\end{array}$ & $\begin{array}{l}0.01 \\
0.60\end{array}$ & $\begin{array}{r}0.22 \\
-0.03\end{array}$ \\
\hline 4. $\mathrm{FH}$ & $\begin{array}{r}g \\
d \times g\end{array}$ & $\begin{array}{l}0.82 \\
0.40\end{array}$ & $\begin{array}{l}0.31 \\
0.11\end{array}$ & $\begin{array}{r}0.09 \\
-0.48\end{array}$ & $\begin{array}{r}-0.12 \\
0.52\end{array}$ & $\begin{array}{l}-0.14 \\
-0.26\end{array}$ \\
\hline 5. GL & $\begin{array}{r}g \\
d \times g\end{array}$ & $\begin{array}{r}0.62 \\
-0.02\end{array}$ & $\begin{array}{l}0.36 \\
0.50\end{array}$ & $\begin{array}{r}0.50 \\
-0.51\end{array}$ & $\begin{array}{l}0.26 \\
0.19\end{array}$ & $\begin{array}{l}0.03 \\
0.04\end{array}$ \\
\hline 6. CW & $\begin{array}{r}g \\
d \times g\end{array}$ & $\begin{array}{l}0.43 \\
0.21\end{array}$ & $\begin{array}{l}0.18 \\
0.45\end{array}$ & $\begin{array}{r}0.59 \\
-0.48\end{array}$ & $\begin{array}{l}0.49 \\
0.20\end{array}$ & $\begin{array}{r}0.10 \\
-0.04\end{array}$ \\
\hline 7. $\mathrm{CN}$ & $\begin{array}{r}g \\
d \times g\end{array}$ & $\begin{array}{l}0.72 \\
0.49\end{array}$ & $\begin{array}{l}-0.10 \\
-0.55\end{array}$ & $\begin{array}{l}-0.34 \\
-0.53\end{array}$ & $\begin{array}{l}-0.49 \\
-0.16\end{array}$ & $\begin{array}{r}0.17 \\
-0.01\end{array}$ \\
\hline 8. SY & $\begin{array}{r}g \\
d \times g\end{array}$ & $\begin{array}{l}0.86 \\
0.52\end{array}$ & $\begin{array}{l}-0.16 \\
-0.54\end{array}$ & $\begin{array}{l}-0.03 \\
-0.32\end{array}$ & $\begin{array}{r}-0.21 \\
0.14\end{array}$ & $\begin{array}{r}0.04 \\
-0.17\end{array}$ \\
\hline $\begin{array}{l}\text { Percentage } \\
\text { of all the pr } \\
\text { over genoty }\end{array}$ & $\begin{array}{l}\text { total variance } \\
\text { l components }\end{array}$ & $27 \%$ & $17 \%$ & $14 \%$ & $11 \%$ & $7 \%$ \\
\hline
\end{tabular}

$\uparrow$ Correlation values less than $|0.5|$ are printed in italics.

first three principal components are correlated with both genotypic and interaction components while the fourth and fifth principal components are most highly correlated with interaction components. The first principal component is fairly highly correlated with the genotypic components of growth rate, leaf length, final height, capsule length, capsule number and seed yield. It also shows a correlation of 0.52 with the interaction component of seed yield. The highest correlation $(0 \cdot 86)$, however, is with the genotypic component of seed yield. Genotypes with an extreme score on this component either have a high average growth rate, leaf length, final height, capsule length, capsule number and seed yield over densities or they have a low average value for these traits over densities. The second principal component is fairly highly correlated in a positive direction with the genotypic component of flowering time. It is negatively correlated, however, with the 
interaction components of growth rate, leaf length, capsule number and seed yield. Genotypes with an extreme score on this component either flower late on average over densities, grow slower under normal density conditions and show a negative or low difference in leaf length, capsule number and seed yield between densities or they flower early on average over densities and show a high positive difference in growth rate, leaf length, capsule number and seed yield between densities (meaning that they perform better under normal density conditions). The third principal component is positively correlated with the genotypic components of capsule width and negatively correlated with the interaction component of capsule number. Genotypes with an extreme score on this component show either a high or low average capsule width over densities associated with a low or high difference, respectively, in capsule number between densities. The fourth principal component is most highly correlated with the interaction components of flowering time, leaf length and final height. Genotypes with an extreme score on this component are found to either flower later, have larger leaves and grow taller under normal density conditions or show a negative or low difference for these traits between densities. The fifth principal component is most highly correlated with the interaction component of flowering time so that genotypes with an extreme score on this component flower either later or earlier under normal relative to high density conditions.

\section{Discussion}

From Sections 4 and 5 it is clear that for experiment 1 most of the genotype-environmental interactions of the traits, FT, GR, LL and FH, which are linearly related to the dependent environmental component, $\hat{\epsilon}_{j}$, are due to the differential responses of the genotypes to successive sowing dates. But it is not always their differential linear responses to successive sowing dates that is responsible as may be especially noted for growth rate. Similarly in experiment 2 it is usually one of the main fertiliser treatments, $\mathrm{N}, \mathrm{P}$ or $\mathrm{K}$, that is responsible although the particular treatment for each trait, FT, GR, LL and FH, may differ according to whether calcium is absent or present. Only the relative magnitudes and not the signs of the correlations between the linear regression coefficients, $\hat{\beta}_{d}$ 's, against $\hat{\epsilon}_{j}$ of the genotypes and their corresponding scores on each of the orthogonal interaction comparisons in table 6 for experiment 1 and table 7 for experiment 2 is of importance. Each regression coefficient, $\hat{\beta}_{d}$, is obtained from the linear regression of the genotype's interactions (without orthogonal breakdown) against the dependent environmental component, $\hat{\epsilon}_{j}$, derived from the mean of all the genotypes in each environment. Negative correlations for certain interaction comparisons of some of the traits in tables 6 and 7 are simply due to the fact that in each environment the average performance of the genotypes is in the opposite direction to the sign of the corresponding orthogonal coefficient, as given in Section 3, of the particular comparison. For those negative correlations which are large in magnitude this is easily confirmed by examining the relative values of the corresponding dependent environmental component, $\hat{\epsilon}_{j}$, in each environment. For any particular trait in each experiment, therefore, the proportion of the variation due to $g \times e$ interactions accounted for by the heterogeneity of regressions against $\hat{\epsilon}_{j}$ is a function of the magnitudes of both the component of variance, $\hat{\sigma}^{2}$ (as given 
in tables 3,4 or 5 ) and of the absolute value of the correlation with $\hat{\beta}_{d}$ (as given in tables 6 or 7) for each interaction cornparison.

In each of tables 3, 4 and 5 each orthogonal environmental component has more or less the same ranking whether based upon the magnitude of its component of variance, $\hat{\sigma}^{2}$, as a main effect or as an interaction with genotypes. This must mean that the genotypes are responding to the same environmental stimuli in the same general direction and this condition must be satisfied in order to detect interactions linearly related to the dependent environmental component, $\hat{\epsilon}_{j}$. For experiment 1 it is found that with successively later sowing dates the 82 inbred genotypes in general flower earlier, have larger leaves and grow taller. The environmental variables responsible are presumably ones which change systematically through the season such as daylength or temperature. Perkins (1972) showed that the two parental varieties 1 and 5 of the 82 inbred genotypes responded in a positive direction for the character final height to changes from season to season in the linear and quadratic derivatives of sowing date, to the linear derivative of a principal component most highly correlated with mean hours of daily sunshine and to both the linear and quadratic derivatives of a principal component correlated with mean daily rainfall (inches) and mean daily minimum temperature $\left({ }^{\circ} \mathrm{F}\right.$.). It is difficult to explain the cubic response of the genotypes in growth rate to successive sowing dates characterised by a general lowering of growth rate in the second sowing in terms of an environmental variable which changes systematically through the season. Neither do simple fluctuations from sowing to sowing in the mean value of certain weather factors such as daily sunshine, rainfall, maximum and minimum temperature and relative humidity over each growing period seem to account for this response. Growth rate must therefore be dependent upon a complex interaction of more than one environmental variable. For experiment 2 it is found that the 10 inbred lines in general flower earlier in the presence of phosphorus (with calcium absent or present), grow faster and taller in the presence of phosphorus if calcium is absent but in the presence of potassium if calcium is present and have larger leaves in the presence of nitrogen without calcium. In this experiment growth rate responds to the same environmental stimuli as final height but Perkins and Jinks (1973) showed that for the same set of 10 inbred genotypes, chosen from among the 82 as a stratified sample for mean final height, the responses of the two characters to the different combinations of sowing dates and planting density in experiment 1 are not associated.

Before discussing the results of the principal components analyses the repeatabilities of the mean performance over environments and of the total interaction with environments for the inbred families will first be given for the traits concerned. The general formula used is

$$
\frac{\hat{D}}{\hat{D}+\frac{1}{n} \hat{E}}
$$

where $\hat{D}$ is an estimate of the additive genetic variance, $\hat{E}$ an estimate of the corresponding environmental variance and $n$ is equal to the number of replicate individuals per genotype multiplied by the number of environments for the repeatability of family means (mean genotypic performance) over environments and to the number of replicate individuals per genotype only 
for the repeatability of the genotype-environmental interactions. The repeatabilities are also really heritabilities since they have been obtained for a population of inbred lines derived without selection from a random sample of individuals of the same $F_{2}$ generation from a cross between two inbred varieties, 1 and 5 , of $\mathcal{N}$. rustica. They are given as percentages in table 11 for the genotypic and genotype-environmental interaction comparisons of FT, GR, LL and FH in both experiments 1 and 2 (in both the absence and

TABLE 11

The heritabilities (as percentages) of the genotypic and genotypeenvironmental interaction, $g \times e$, components of the traits involved in the principal components analyses (see Section 2 for an explanation of the abbreviations)

\section{Experiment 1}

Sowings 1, 2, 3 and 4 each with ND and HD

$\begin{array}{lcc} & \text { Genotypic } & g \times e \\ \text { FT } & 99 & 54 \\ \text { GR } & 98 & 65 \\ \text { LL } & 97 & 25 \\ \text { FH } & 99 & 55\end{array}$

$\begin{array}{lcc}\text { CL } & \begin{array}{c}\text { Sowing } 1 \text { only with } \\ \text { ND and HD }\end{array} \\ \text { CW } & 93 & 23 \dagger \\ \text { CN } & 92 & 25 \dagger \\ \text { SY } & 93 & 56 \\ \text { Genotypic } & 91 & 47\end{array}$

\section{Experiment 2}

Calcium absent

$\begin{array}{lccccc} & \overbrace{\text { Genotypic }} & g \times e & & \overbrace{\text { Genotypic }} & g \times e \\ \text { FT } & 99 & 50 & & 99 & 47 \\ \text { GR } & 99 & 70 & 99 & 56 \\ \text { LL } & 93 & 27 & 96 & 8 \dagger \\ \text { FH } & 99 & 68 & 99 & 70\end{array}$

$\dagger$ Genotype-environmental interactions non-significant.

presence of calcium for the latter experiment) and for CL, CW, CN and SY in the two planting densities of sowing 1, experiment 1 . The components of variance, $\hat{\sigma}^{2}$, of the genotypic or $g \times e$ interaction comparison was used in each case as an estimate of $D$ and the corresponding error variance as an estimate of $E$. It is clear from the table that the repeatability of the genotypic comparison is consistently high for all the traits whereas that of the corresponding $g \times e$ interaction comparison is usually much lower. In general terms, therefore, the contribution to the principal components by the genotypic comparisons will be mostly genetic in origin whereas that by the $g \times e$ interaction comparisons will be partly genetic and partly environmental in origin. The problem of having, in multivariate statistical analysis, an environmental contribution to the genetic covariation of family means is discussed by Gale and Eaves (1972).

The correlations (over genotypes) between the score of each genotype on the first principal component and the corresponding scores on each of the genotypic and $g \times e$ interaction components of FT, GR, LL and FH in experiment 2 , in both the absence and presence of calcium, were as anticipated (table 9) and do not require further discussion. Most of the interactions of FT, GR, LL and FH in experiment 1 are with the linear, quadratic or cubic effects of sowing dates. Not many of the principal components, 
therefore, are expected to be shared in common between the analysis of FT, GR, LL and FH in experiment $\mathrm{l}$ and of FT, GR, LL, FH, GL, GW, GN and SY in just the two planting densities of sowing 1 unless they are most highly correlated with genotypic comparisons. Thus the same genotypes tend to have extreme scores on the first principal component of the four primary traits in experiment 1 and on the second principal component of the four primary and four fitness traits in sowing 1 and both components are most highly correlated with the genotypic component of flowering time. In a similar manner the fourth principal component of the four primary traits in experiment 1 can be matched with the first principal component of the four primary and four fitness traits in sowing 1 and both components are fairly highly correlated with the genotypic component of leaf length (see tables 8 and 10).

The first principal component of the four primary and four fitness traits in the two planting densities of sowing $l$ is most highly correlated with the genotypic component of seed yield (table 10). It is also quite highly correlated with the genotypic components of all the other characters except those of flowering time and capsule width. The fitter genotypes, defined as those having a higher average seed yield per plant, therefore also tend to grow faster, have longer leaves, grow taller, have longer capsules and a larger number of capsules on average over densities. Jinks (1954) has similarly reported a high correlation between the number of capsules per plant and final plant height for a diallel set of crosses between eight diverse inbred varieties of $\mathcal{N}$. rustica. Biometrical genetical interpretations of the fitness traits based upon information from not just the 82 inbred lines but also from their ancestral parental (inbred varieties 1 and 5) and $F_{1}$ generations will be given in a future paper. The eight characters (FT, GR, LL, FH, CL, GW, GN and SY) are unusually combined in the least fit genotype, number 70. Thus this genotype flowers extremely late and has a low average performance for all the other characters including final height. It also has an extreme score on principal components 1 and 3 of the four primary traits in experiment 1 and on components 1 and 2 of the four primary and four fitness traits in sowing 1.

Interpretation of the second principal component given in table 10 for the eight characters in the two planting densities of sowing 1 shows that those genotypes which on average flower earlier over the two densities tend to suffer more in growth rate, leaf length, capsule number and seed yield under high density conditions while those genotypes which on average flower later over densities tend to actually grow faster under high density conditions and show a low difference in leaf length, capsule number and seed yield between densities. From this it can be inferred that the development of the earlier flowering genotypes is retarded in certain respects when the availability of environmental resources is limited by the increased competition at the higher density.

For FT, GR, LL and FH in experiment 1 the first four principal components account for less than half (45 per cent) of the total standardised variance of the traits over genotypes. However, meaningful albeit complex interrelationships between the genotypic and $g \times e$ interaction components of the traits are revealed. These components have been derived within a trait as a set of orthogonal comparisons so that they are statistically independent and any associations detected by principal components analysis are 
most likely to be genetic in origin. Close linkage or pleiotropy may be responsible. Since the genes have had a number of opportunities to recombine from the $F_{1}$ to $F_{9}$ generations loose linkage between them is unlikely to be the cause. The genotypic components of flowering time and final height were both positively associated with the first principal component, that of growth rate with the second component and that of leaf length with the fourth. For the same set of inbred lines grown in 1970 in just two blocks Eaves and Brumpton (1972) were able to summarise the eight primary genotypic factors from a factor analysis into two second-order factors, one related to flowering time and growth and the other to leaf morphology. The $g \times e$ interaction components whose scores were most highly correlated with those of the principal components over genotypes are also those found to be primarily responsible for the heterogeneity of regressions of the total interactions of the genotypes against the dependent environmental component, $\hat{\epsilon}_{j}$. They are not observed to be strongly associated with the genotypic component of the same trait. Perkins and Jinks (1973) similarly reported a non-significant correlation between the linear regression coefficient, $\hat{\beta}_{d}$, of the interactions of each genotype against $\hat{\epsilon}_{j}$ and its corresponding additive genetic component $[\hat{d}]$, for each of the characters except flowering time. They can, however, show a complex association with the genotypic component of another trait (table 8 ). The strong association between the genotypic and important $g \times e$ interaction components of FT, GR, LL and $\mathrm{FH}$ with the first principal component in both the absence and presence of calcium is due to the selection of the genotypes and the expected convergence of quantitative traits in poorer sets of environments (Perkins and Jinks, 1973). The most productive application of the principal components analysis was to the four primary and four fitness traits in sowing 1 , experiment 1 , for which the first five components accounted for 76 per cent of the total standardised variance of the traits over genotypes. The first principal component was highly correlated with the genotypic component of seed yield and also with that of several other traits whereas the second component was most highly correlated with the genotypic component of flowering time and negatively correlated with the interaction component between densities and genotypes of several of the traits including that of capsule number and seed yield.

Acknowledgments.-These are due to Professor J. L. Jinks for his most helpful advice on the manuscript and to Birmingham University for the use of their KDF-9 Computer.

\section{RefERENGES}

EAVES, L. J., AND BRUMPTON, R. J. 1972. Factors of covariation in Nicotiana rustica. Heredity, 29, 151-175.

Fisher, R. A., AND yates, F. 1963. Statistical Tables for Biological, Agricultural and Medical Research. Oliver and Boyd, London.

FREEMAN, G. H., AND PERKINs, JEAN M. 1971. Environmental and genotype-environmental components of variability. VIII. Relations between genotypes grown in different environments and measures of these environments. Heredity, 27, 15-23.

Gale, J. S., AND EAves, L. J. 1972. Variation in wild populations of Papaver dubium. V. The application of factor analysis to the study of variation. Heredity, 29, 135-149.

JiNks, J. L. 1954. The analysis of continuous variation in a diallel cross of Nicotiana rustica varieties. Genetics, 39, 767-788. 
LINNEY, R., BARNES, B. W., AND KEARSEY, M. J. 1971. Variation for metrical characters in Drosophila populations. III. The nature of selection. Heredity, 27, 163-174.

PERKINS, JEAN M. 1972. The principal component analysis of genotype-environmental interactions and physical measures of the environment. Heredity, 29, 51-70.

PERKINS, JEAN M., AND JINKS, J. L. 1968. Environmental and genotype-environmental components of variability. III. Multiple lines and crosses. Heredity, 23, 339-356.

PERKINS, JEAN M., AND JINKs, J. L. 1973. The assessment and specificity of environmental and genotype-environmental components of variability. Heredity, 30, 111-126.

SEAL, H. 1964. Multivariate Statistical Analysis for Biologists. Methuen, London.

WESTERMAN, JANE M., AND LAWRENCE, M. J. 1970. Genotype-environment interaction and developmental regulation in Arabidopsis thaliana. Heredity, 25, 609-627. 